\title{
ANNOUNCEMENT: RADIOCARBON LABORATORY QUALITY ASSURANCE PROGRAM (SIRI)
}

The issue of comparability of measurements (and ultimately bias, accuracy, and precision of measurement) is one that has been the focus of some attention both within the ${ }^{14} \mathrm{C}$ community and the wider user communities. The design and organization for the sixth (SIRI) radiocarbon laboratory intercomparison is intended to complement and extend previous radiocarbon international quality assurance programs run successfully by Glasgow University and SUERC, most recently TIRI (1991-1995), FIRI (1997-2002), and VIRI (2004-2008), with results published in the Radiocarbon journal (Scott 2003; Scott et al. 2010a,b).

The aims and objectives of SIRI are:

- to demonstrate the comparability of routine analyses carried out in radiocarbon laboratories;

- to quantify the extent and sources of variation in results;

- through choice of material to contribute to the discussion concerning laboratory offsets and error multipliers in the context of IntCal (the International Calibration program);

- to gain a better understanding of differences in background derived from a range of infinite-age material types.

SIRI will be a single-stage proficiency trial, lasting approximately 2.5 years. Samples will include a sequence of tree rings, and several background samples suitable for AMS analysis. While it will not be possible to provide the same samples for radiometric laboratories, a small, distinct set of samples will be available for LSC and GPC laboratories.

Samples will be distributed in early 2013 .

If you wish to participate or wish further information, please send your contact details to Prof. Marian Scott, marian.scott@glasgow.ac.uk.

The financial support of English Heritage is gratefully acknowledged.

Marian Scott, Gordon Cook, Phil Naysmith

Glasgow, July 2012 